\title{
Current concepts and principles in open tibial fractures - part ii management
}

\begin{abstract}
Throughout history, there has been a fear of open tibial fractures due to the high incidence of infection potentially leading to amputation and death. Before the development of standardized surgical wound treatment and antisepsis, amputations were frequently required to prevent spread of sepsis and risk of death. Even today, this remains a challenging condition to treat. In recent years efforts have been made to develop new techniques and to promote new evidence based guidelines for the management of these injuries. The cooperation between orthopaedic and plastic surgeons together with the use of modern surgical instruments and techniques and modern wound management has resulted in a dramatic reduction in infection rates in many parts of the developed and developing world.
\end{abstract}

Keywords: Review, Open fracture, Tibia, Managemen
Volume 8 Issue 2 - 2017

\author{
Fabian L Poletti,' Simon Macmull, ${ }^{2}$ Nadeem \\ Mushtaq, ${ }^{2}$ Reza Mobasheri ${ }^{2}$ \\ 'Dorset County Hospital Foundations Trust, UK \\ ${ }^{2}$ Imperial College Healthcare NHS Trust, UK
}

Correspondence: Fabian Poletti MD, MSc, Orthopaedics and Trauma Surgery, Dorset County Hospital NHS Foundation Trust, UK Email fabypoletti@hotmail.com

Received: March 17, 2017| Published: April 24, 2017

\section{Introduction}

Open tibial fractures are challenging injuries to treat. There has been a fear of open tibial fractures due to the increased propensity of infection that has the potential of resulting in amputation or even death. Prior to the advancement of standardized surgical wound treatment and antisepsis and antibiotics, mutations were often a mainstay in the prevention of overwhelming sepsis and ultimately death. Even today, this remains a challenging condition to treat. Recently efforts have been made to develop new techniques and to promote evidence based guidelines for the management of these injuries. The co-operation between orthopaedic and plastic surgeons together with the use of modern surgical techniques and wound management has resulted in a reduction in infection rates.

\section{Management}

\section{Principles}

In their original paper Gustilo and Anderson stated "There is universal agreement that open fractures require emergency treatment, including adequate debridement and irrigation of the wound".' revention of wound sepsis remains the prime objective in the management of open fractures. The reported infection rates in these fractures, which range from 3 to $25 \%$, are a challenge to every surgeon who treats them. ${ }^{2}$ The actual principles for treating an open fracture include: resuscitation, initial recognition of the severity of the injury, analgesia, anti-tetanus therapy, antibiotics therapy; debridement and irrigation, rigid stabilization of the fracture, possibly use of adjuncts such as antibiotic beads and vacuum therapy, and early soft tissue coverage with well vascularized tissue. The use of antibiotic beads and vacuum therapy are not mandatory, but are an important part of the armamentarium for those caring for such fractures.

\section{Resuscitation}

It is important to remember that the fracture is part of a pathological process that is happening to the patient who has sustained injury as the result of a low or high-energy trauma. Therefore the first priority will always be to save the life over limb of the patient using the principles of the Advanced Trauma Life Support . ${ }^{2}$ When the patient is stable the next priority is to save the limb where there fracture is located. When the survival of the limb is secured we need to think what needs to be done to preserve the function of that limb, as saving a limb without a good function can be much worse than having an early amputation and limb fitting rehabilitation.

\section{Initial recognition of the severity of the injury}

In the majority of cases the recognition of the severity of the injury may be obvious, but the degree of tissue damage can be underestimated. It is important to be aware of closed degloving or crush injuries. One must be watchful of vessel and nerve injury and early involvement of senior orthopaedic and plastic surgeons is the key to management. It is important to take photographic documentation and to cover the wound appropriately. ${ }^{2}$ According to the British Orthopedic Association and British Association of Plastic, Reconstructive and Aesthetic Surgeons (BOA/BAPRAS) guidelines "Standards for treatment of open fractures of the lower leg" an incomplete debridement of the open fracture in Accident and emergency yields little benefit. ${ }^{3}$ Exploration of the wound in the Emergency Department should be avoided as it has not shown any advantage. In fact lavage of the open wound can result in further dissemination of the debris into open tissues. ${ }^{2}$ Wound management in the Emergency Department should consist of removal of gross contamination, agricultural or marine waste. A photograph of the wound should be taken so that the wound can remain covered and there is documentation of the wound. Moistened dressings with normal saline should be used to cover the wound and an adhesive dressing to seal it. Antiseptic soaked dressings should be avoided. The limb should be immobilized on the patient's arrival to hospital. The splint should be of an appropriate size and of sufficient length and adequately applied. The splint should allow for regular examination of the neurovascular status of the limb. ${ }^{2}$ Simple radiographs complete the assessment. Normally two orthogonal radiographs that incorporate the joints above and below are adequate, however, more images or even Computed Tomography scans (CT), are sometimes necessary to obtain sufficient radiological assessment. Accepting inadequate views is a common reason for missed injuries and can result in affecting the surgical outcome. ${ }^{2}$

Analgesia: It is important to offer the patient good pain relief.

Anti-tetanus therapy: This should be provided as a matter of routine if the patient is not up to date with vaccinations or unsure/obtunded.

Antibiotics prophylaxis: Approximately $24-70 \%$ of open fractures are contaminated with bacteria. ${ }^{3}$ If antibiotic coverage is not administrated, the infection rate may be high. However the exact 
type, doses and time of administration of the antibiotics depends of the type of the injury and the protocol used in the local hospital. There is strong evidence for the efficacy of first generation cephalosporins in the management of open fractures. ${ }^{2}$ The BOA/BAPRAS guidelines, however, advise avoidance of cephalosporins because of the apparent association with Clostridium Difficult. The BOA/BAPRAS suggests that antibiotic prophylaxis should be driven by advice from microbiology and antibiotics used should have a broad spectrum of activity that is similar to cephalosporins. Regarding the doses and the specific antibiotics, BOA/BAPRAS guidelines advise the following principles. ${ }^{4}$

1. The antibiotic of choice is co-amoxiclav ( $1.2 \mathrm{~g} / 8$ hourly), or a cephalosporin (e.g. cefuroxime $1.5 \mathrm{~g} / 8$ hourly), and this should be continued until first debridement (excision).

2. At the time of first debridement, co-amoxiclav (1.2 $\mathrm{g}$ ) or a cephalosporin (such as cefuroxime $1.5 \mathrm{~g}$ ) and gentamicin (1.5 $\mathrm{mg} / \mathrm{kg}$ ) should be administered and co-amoxiclav/cephalosporin continued until soft tissue closure or for a maximum of 72 hours, whichever is sooner.

3. Gentamicin $1.5 \mathrm{mg} / \mathrm{kg}$ and either vancomycin $1 \mathrm{~g}$ or teicoplanin $800 \mathrm{mg}$ should be administered on induction of anaesthesia at the time of skeletal stabilisation and definitive soft tissue closure. These should not be continued post-operatively. The vancomycin infusion should be started at least 90 minutes prior to surgery.

4. Patients with anaphylaxis to penicillin should receive clindamycin (600mg iv pre-op/qds) in place of co-amoxiclav/cephalosporin. For those with lesser allergic reactions a cephalosporin is considered to be safe and is the agent of choice.

Evidence suggests that antibiotic should be administered as soon as possible following the index injury, ideally within three hours of the contamination. ${ }^{4}$ As debridement, skeletal stabilization and definitive soft tissue coverage is unlikely to occur on the same day as the patient is admitted the guidelines indicate the importance of antibiotic prophylaxis.

\section{Wound debridement and irrigation}

Gustilo states "Adequate debridement is the single most important factor in the attainment of a good result in the treatment of an open fracture". ${ }^{1}$ With the patient in the Operating Room, a systemic debridement must be performed with the removal of gross contamination and debris, from superficial layers to deep structures. The Use of tourniquet should be minimized. ${ }^{2}$ All the necrotic tissue should be excised, with a wound extension for full evaluation of the soft tissue injury. As Gustilo states "The muscle viability is determined by the four C's of Scully: contractility, color, consistency and capacity to bleed". ${ }^{1}$ All muscular tissue that does not meet these criteria should be excised. On evaluation of the bone, all free periosteum and any completely free cortical fragments need to be excised. When the determination of viability is inconclusive at the time of the initial debridement, repeated debridements at 24-48 hour intervals can be employed to eliminate devitalized tissue (second look surgery). ${ }^{2}$ Good irrigation is fundamental to supplement a systematic debridement in removing foreign material and decreasing the bacterial load. Gustilo stated "Copious irrigation implies that the wound is irrigated with normal saline solution in large amounts in conjunction with debridement in order to remove all dirt and foreign material as well as all devitalized tissue". ${ }^{1}$ The amount of the irrigation depends of the type of the fractures. Draeger R W et al. ${ }^{5}$ al found on assessment of Gustilo-Anderson grades that in type 1 fractures, 3 litres of saline irrigation decreases infection rates, 6 litres in type 2 fractures and 9 litres in type 3 fractures. ${ }^{5}$ According to Angeln et al. ${ }^{6}$ antiseptic or antibiotic solutions have not been shown to decrease infection rates and they have been linked to tissue damage and thus should be avoided. ${ }^{6}$ Non-sterile soaps show the same effectiveness, less tissue damage and are more economical. ${ }^{6}$ Several studies have shown pulse lavage to be detrimental to bone and soft tissues, can slow bone healing and potentially drive bacteria further into the wound. ${ }^{2-}$ ${ }^{11}$ Therefore the use of pulsatile lavage systems is unwarranted. A simple brush-suction irrigation method plus bulb syringe irrigation removes more contaminant than HPPL whilst causing less tissue damage..$^{2-11}$ Recommendations over the last century for open fractures have been emergent irrigation and debridement within 6 hours. ${ }^{2}$ These timing recommendations are likely derived from the pre-asepsis war era, data on bacterial doubling times, and limited animal studies. The BOA/BAPRAS guideline stated "The origin of this 6 hours rule remains unclear; it is often quoted but is largely unreferenced". Several studies that call into question the urgency of open fracture care exist. ${ }^{12,13}$ A recent meta-analysis evaluated how timing to debridement affects infectious complications in open long bone fractures. They concluded that there was no association between delayed debridement and higher infection rates. ${ }^{12,13}$ Skaggs and associates reviewed 554 open fractures in children and found only an association of time of antibiotic administration with decreases in infection rates. Debridement was performed as late as 24 hours post-injury. In another study, Noumi et al. reviewed 88 patients and examined factors contributing to deep infection after IM nailing of open long bone fractures. Only the fracture grade was consistent with increased infection risk. The fracture classification was predictive of nonunion. However, no association was found between timing of debridement and the risk for infection or nonunion. ${ }^{12}$ Harley and colleagues reviewed 241 long bone fractures and recorded transport time from the field to hospital admission, which was frequently in excess of 8 hours. A weak association between debridement time after 13 hours and infection was found. ${ }^{12,13}$ The role of extended transport time and delay in antibiotics was not explored. Data from the Lower Extremity Assessment Project Group (LEAPG), a multicenter limb salvage study, yielded 315 open fractures. After multivariate analysis, only time of injury to admission was discovered to correlate with infection risk. Time to debridement was not predictive of infection. The investigators cited resuscitation and early administration of broad spectrum antibiotics, or either, as possible reasons for the importance of admission time. ${ }^{12,13}$ Most studies conclude that time to debridement is not an independent predictor of deep infection for open fractures. ${ }^{2-13}$ Of greater importance is time to admission and the early administration of antibiotics. The timing of debridement should therefore be determined by:

\section{a. adequacy of resuscitation}

\section{b. presence of appropriate and alert staff and OR availability}

Even if Gustilo et al. ${ }^{1}$ in their original paper defined open fractures an as emergency, open fractures should be considered with urgency rather than as an emergency in the absence of limb-threatening injury. ${ }^{2}$ Although no Level I data exists, it is unlikely that the quality of data will improve, as there are numerous uncontrollable factors preventing such a study.

\section{Rigid stabilization}

At the time of the initial wound debridement skeletal stabilization is essential. ${ }^{2,14}$ It provides bony alignment and reduces gross movement of the of the bone ends. This therefore prevents further injury to the soft tissue envelope and preserves blood supply. It also has a positive effect on control of pain and limb oedema by improving perfusion and 
venous return of the affected limb. ${ }^{2-19}$ The BOA/BAPRAS guidelines advocates the use of the spanning external fixator, however care needs to be taken on applying the external fixator as access to the wounds by the plastic surgeons is required for soft tissue reconstruction. (Figure 1) The safe corridors for pin placement in the tibia should be familiar for the orthopaedic surgeon who is performing this procedure. ${ }^{14}$ Controversy remains regarding the mode of stabilization that should be used in open fractures. Numerous studies have concluded that external fixators are $\mathrm{c}$ the preferred method for obtaining bone stability. ${ }^{2,13}$ The advent of reamed locking nails was initially met with caution. There were concerns that reaming would result in compromise of the intramedullary blood supply and possibly the cortical blood supply resulting in non-union and increased infection risk. ${ }^{2,13}$ The introduction of unreamed locking nails sparked new interest in the treatment of open fractures as there was the belief that blood supply would not be compromised to the same extent. The development of Minimally Invasive Plate Osteosynthesis (MIPO) led to designs of plates that were design of contoured implants which would cause a more limited interruption of the periosteal blood supply and have been found to have a role in the treatment of open fractures even of the tibia. ${ }^{13}$ According to the $\mathrm{BOA} / B A P R A S$ guidelines the degree of bone loss and the morphology of the fracture pattern will guide the most appropriate form of definitive skeletal fixation. ${ }^{2}$ (Table 1).

Table I Rigid Stabilization for open tibia fractures. BOA/BAPRAS guidelines

\section{BOA/BAPRAS Guidelines Regarding Rigid Stabilization in Open}

\section{Tibia Fractures}

The use of spanning external fixation when definitive stabilization and immediate wound cover is not carried out at the time of primary debridement Exchange from spanning external fixation to internal fixation must be done as early as possible

Internal fixation is safe if there is minimal contamination and soft tissue coverage is achieved at the same time as insertion of the implant

Modern multi-planar and circular fixators must be used if there is significant contamination, bone loss and multilevel fractures of the tibia

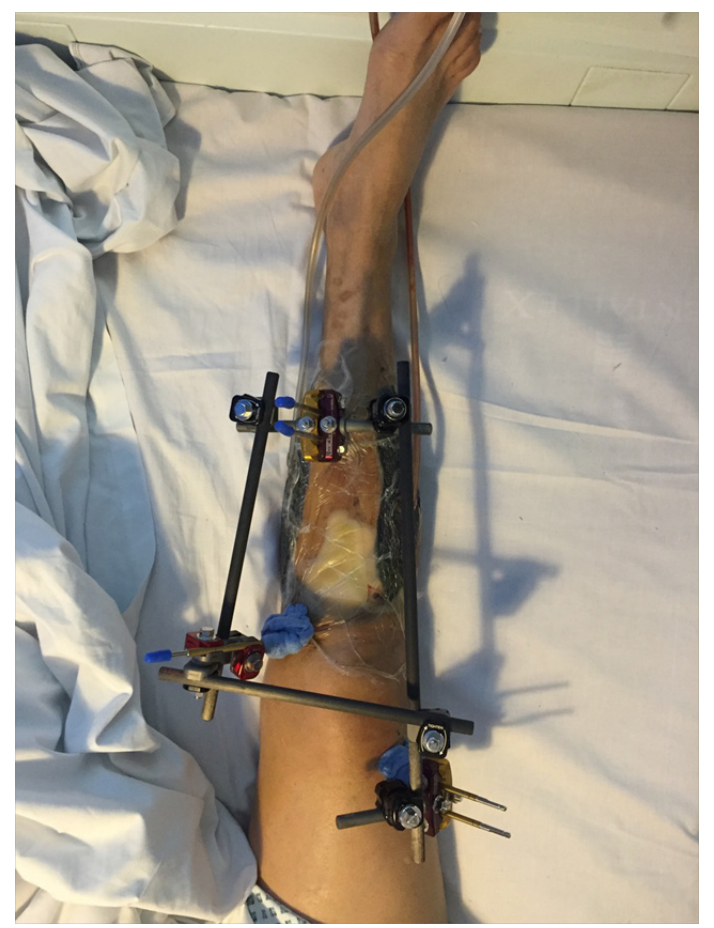

Figure I Spanning External fixator for a tibia open fracture after initial debridement. Imperial College Healthcare NHS Trust, St Mary's Hospital, London. United Kingdom.
External fixator: The management of open fractures using external has been in and out of fashion over the past one hundred years and more. Vidal and Andrey reignited interest in the use of external fixators and proved that their use allowed for easy access to the wounds as well as providing adequate stabity even for the most comminuted of fractures. In a recent meta-analysis conducted by Giannoudis et al. ${ }^{14}$ a total of 536 open tibia fractures were treated by external fixation, of which $82 \%$ were grade III open injuries. ${ }^{14}$ The group found that they managed to achieve bony union in $94 \%$ of their patient group at a mean time of 37 weeks. Soft tissue coverage was delayed for more than 72 hours in all the patients treated. Severe open tibial fractures frequently require repeated procedures such as soft tissue coverage, exchange nailing and bone grafting to achieve bony union. The Giannoudis study noted a significant malunion rate of around $20 \%$ in their study group. The incidence of deep infection was $16.2 \%$, with $4.2 \%$ developing chronic osteomyelitis. Pin-track infection was recorded at up to $32.2 \% .^{14}$

Unreamed intramedullary nails: Giannoudis's meta-analysis reviewed 666 fractures, with over half graded Gustillo Anderson III. ${ }^{14}$ They found that union was achieved in $96 \%$ of those that received an undreamed nail as skeletal stabilization. They noted that there was an increased rate of fatigue failure of the smaller diameter undreamed nails of $12.4 \%$. Infection rates were recorded as deep infection of $7 \%$, osteomyelitis in $0.7 \% .33 \%$ of patients required at least one further surgery. Delayed union was a common finding as would be expected with significant injury to the soft tissue envelope and was seen in $22 \%$ of patients.

Reamed intramedullary nails: Giannoudis et al. ${ }^{14}$ also studied the use of reamed nails in the treatment of 187 open injuries. $43 \%$ of which was grade-III open injuries. ${ }^{14}$ They found that union rates were slightly improved compared to the undreamed nail group with union noted in $97 \%$. Deep infection was recorded in $6.4 \%$, with similar rates of chronic osteomyelitis as seen in the undreamed group. At least one further surgery was indicated in $31.6 \%$ for management of delayed bony union or other complication. Implant fatigue failure was only seen in $3 \%$.

External fixation and delayed reamed intramedullary nailing: Some studies have suggested that the management of type III Gustillo-Anderson open fractures should be managed with staged initial external fixation followed by delayed intramedullary nailing to prevent the potential risk of infection with early intramedullary nailing. ${ }^{2,13}$ Giannoudis' analysed this group of patients treated in this manner. Union was achieved in $92 \%$ of patients with a mean time to union of 38.5 weeks. ${ }^{14} 23 \%$ of patients required at least one further surgery. The external fixator was maintained an average of 39 days with a pin track in infection rate of $15.3 \%$. The average time to definitive nailing was 26 days once pin sites had settled and normal inflammatory markers. Infection remained a concern in this group with deep infection rate of $17 \%$ and chronic ostepmyelitis in $2.5 \% .{ }^{14}$ As a result the $\mathrm{BOA} / B A P R A S$ guidelines have recommended that if provisional external fixation is considered and used in conjunction with delayed wound closure the conversion to definitive stabilization should be proceeded to with caution. ${ }^{2}$ Their recommendations include early stabilization with simultaneous soft tissue coverage in tandem with the plastic surgical team. BOA/BAPRAS guideline recommends that this needs to be achieved within 72 hours of the initial debridement and temporary skeletal stabilization. Definitive soft tissue cover should be achieved in the same surgical sitting. Should this not be feasible infection risks significantly increase and definitive skeletal management of with modern multi planar/circular external fixators should be considered. ${ }^{2}$ (Figure 2). 


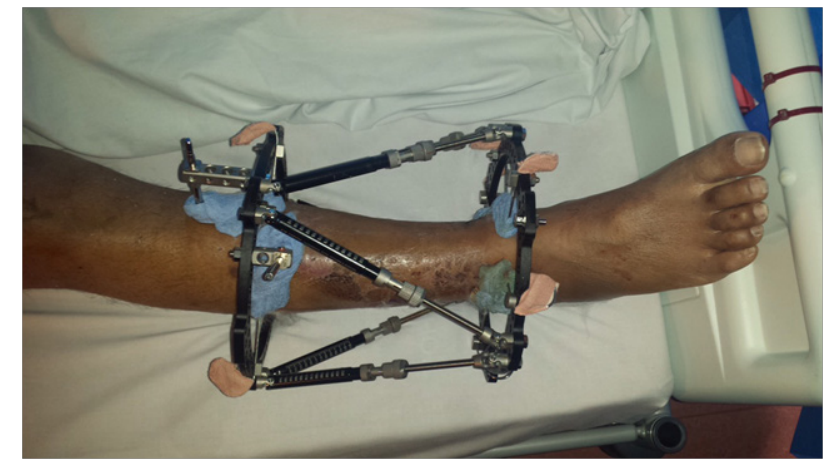

Figure 2 Circular frame: Taylor Spatial frame. Imperial College Healthcare NHS Trust, St Mary's Hospital, London. United Kingdom.

\section{Plates and Screws}

Little evidence exists regarding the use of plating techniques in the skeletal stabilization of open fractures. Giannoudis meta-analysis reviewed comparison of a total of 30 patients treated with external fixation versus 26 treated by plates and screws. ${ }^{14}$ The analysis revealed an increased rate of deep infection rates but not showing any statistical significance. Significant numbers did however exhibit delayed union. Deep infection was higher in those treated with plate and screws but again showed no evidence of statistical significance. Reoperation rate was significantly highgr in the plated group (69\%) compared to those treated with external fixator $(30 \%))^{14}$

\section{Comparison of different types of fixation}

When comparing the use of external fixation vs. unreamed intramedullary nailing Giannoudis et al. ${ }^{14}$ found no statistically significant difference with management of open fractures using external fixation versus unreamed intramedullary interlocking nails. His group no difference between rates of infection and union rates and time to union. ${ }^{14}$ Management with external fixation was found to be associated with statistically significant rates of malunion compared to nailing techniques. Ultimately the analysis showed no evidence of external fixation being a superior method over intramedullary nailing in the treatment of open fractures even in cases with GustilloAnderson injuries up to grade IIIb. ${ }^{14}$ In another meta-analysis, conducted by $\mathrm{Xu}$ et al. ${ }^{15}$ the patients undergoing intramedullary nailing had a lower incidence of postoperative infection, malunion, nonunion, and less healing time compared with external fixation. However, regarding soft tissue injury and delayed healing, there was no significant difference between both methods..$^{15,16}$ These results are in line with previous meta-analysis and studies conducted by Bhandari et al..$^{9}$ and Inan et al. ${ }^{17} \mathrm{BOA} / B A P R A S$ guidelines indicate that in the situation where skeletal stabilization is achieved using internal fixation is used, definitive soft tissue cover must be achieved at the same surgical procedure. ${ }^{2}$ They go on to clarify this by stating that internal fixation is appropriate in the scenario of adequate primary surgical debridement with little wound contamination with soft tissue coverage of the open fracture. Soft tissue coverage can be attained by tension free suturing or local/free musculocutaneous flap and still achieve low infection rates. ${ }^{2}$ Significantly higher rates of infection are seen with delayed soft tissue coverage over internal fixation. The guidelines indicate highly contaminated wounds should avoid treatment with internal fixation. The management of bony injuries associated with significant bone loss or involving articular surfaces or with comminution/complex multi-level fractures should be treated with circular external fixators. External fixators should also be used in association with fractures/dislocations that exhibit significant instability as seen especially around the knee and ankle. ${ }^{2}$ Ultimately the management should be multidisciplinary with a true combined dedicated ortho-plastic approach to achieve the most successful outcome for the patient. ${ }^{2}$

\section{Vacuums Therapy and Antibiotic Beads}

The availability of negative pressure wound therapy using reticulated open cell foam (NPWT/ROCF) as delivered by V.A.C. Therapy has dramatically changed the treatment of soft tissue injuries in orthopaedic trauma patients. ${ }^{2,20}$ (Figure 3). The BOA/ $B A P R A S$ guidelines suggest that the use of negative pressure foam dressings results in reduced injury to the exposed tissue both bone and soft tissue and prove to be at least equivalent to plain soaked gauze dressings. Adequate debridement and lavage of the wound must be performed before these dressings are applied. However they are not substitute for effective wound debridement and lavage. They may have a role in treating limited defects, especially in patients with significant comorbidities but not appropriate for the treatment of larger defects requiring soft tissue graft transfer surgery. ${ }^{2}$ The OTAOFC can be useful in addressing the necessity of Vacuum Therapy and Antibiotic Beads. A recent study shows that skin injury was the only OTA-OFC variable significantly related to VAC use. ${ }^{4}$ The strongest potential predictor of vacuum-assisted closure use is the severity of skin injury, whilst bone loss is the strongest predictor of antibiotic bead placement. ${ }^{4}$ The use of antibiotic beads reduces bacterial load of an open fracture delivering a high doses of antibiotic locally. ${ }^{2}$ Heat resistant antibiotics must be used to accommodate for the exothermic reaction seen when the polymethyl methacrylate cement cures, as it is introduced into the wound cavity, and the area is covered with a semipermeable membrane. The use of localized antibiotic in conjunction with systemic antibiotics has shown to infer lower infection rates than if systemic antibiotics are used alone. ${ }^{2}$

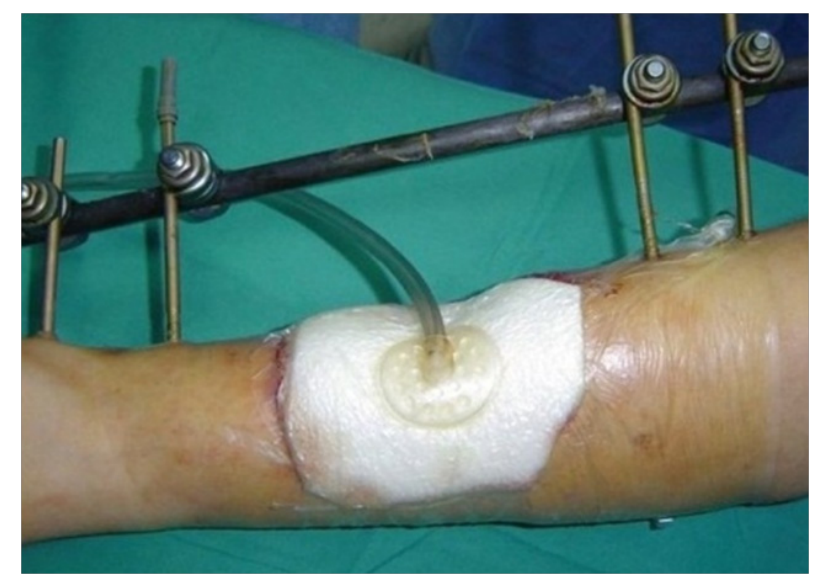

Figure 3 VAC system applied on the wound in a tibia open fracture. Imperial College Healthcare NHS Trust, St Mary's Hospital, London. United Kingdom.

\section{Early soft tissue coverage with well vascularized tissue}

Soft tissue coverage and wound closure helps promote revascularization of injured bone and soft tissues, and prevents late infections and nonunion that may occurs secondary to persistent bone ischemia. ${ }^{15}$ Soft-tissue defect coverage of the lower limbs especially the tibia is a frequently performed procedure due to the increase incidence of "high energy" traumas which affect this location. The $\mathrm{BOA} / B A P R A S$ guidelines advocate early definitive soft tissue cover in the presence of an open fracture. Ultimately the evidence available suggests that following these guidelines would result in improved rates of successful soft tissue grafting and lower rates of deep infection and 
osteomyelitis. ${ }^{2}$ It would seem that immediate soft tissue reconstruction with bony stabilization is the key however the BOA and BAPRAS add the caveat that surgery should be performed by both dedicated Orthopaedic and Plastic Surgeons in specialists units experienced in the management of open fractures. If necessary, surgery should be performed under elective/semi-elective conditions despite the fact that as the soft tissue injury ages the tissues become more difficult to handle as they become more oedematous and friable. ${ }^{2}$ Therefore, the guidelines recommend soft tissue cover should be achieved within the first week of the initial traumatic insult. The type of flap is chosen on the basis of anatomical considerations, specifically the location of the defect on the leg, the size of the defect, and the availability of local tissues for coverage. The BOA/BAPRAS guidelines state the following principles:

a. All open fractures need to be covered with vascularized soft tissue

b. Dressings such as those using foam with negative pressure (VAC) can temporize following wound excision but are not to be used as a substitute for definite flap coverage.

c. Relatively low energy tibial fractures need to be covered by local fasciocutaneus flaps so long as the vascularity has not been compromised by the zone of injury and degloving.

d. Strong clinical evidence to support the use of one form of soft tissue cover over another in open tibia shaft fractures is absent. However, available experimental data would suggest that diaphyseal tibial fractures with periosteal stripping are best covered by muscle flaps instead of fasciocutaneus flaps.

e. Metaphyseal fractures, especially around the ankle, are best covered by fasciocutaneus flaps, including free flaps.

As mentioned earlier better results are seen when patients are treated in dedicated trauma units with the appropriate level of training of staff. Better results are even seen in those with co-morbidities that would have a detrimental effect on wound healing such as peripheral vascular disease and diabetes mellitus. ${ }^{2}$

\section{Limb salvage/amputation}

In contrast to the open fracture classifications, several scoring systems were developed which emphasized surgical decision making with limb-threatening injuries when deciding between limb salvage and amputation. These include the Mangled extremity severity score (MESS); the predictive salvage index (PSI); the Limb Salvage Index (LSI); the Nerve Injury, Ischemia, Soft tissue injury, Skeletal injury, Shock and Age of patient (NISSSA) score; the Ganga Hospital Open Injury Score and the Hannover fracture scale-97 (HFS-97). ${ }^{21,22}$ Unfortunately, the data regarding the management of the mangled extremity are conflicting, and the literature is without Class I studies. ${ }^{23}$ The Lower Extremity Assessment Project group (LEAP) has generated important data raising many questions about indications for immediate amputation with mangled limbs. Lower-extremity injury severity scores at or above the amputation threshold should be cautiously used by a surgeon who must decide the fate of a lower extremity with a high-energy injury. New insights, therapies and techniques will improve outcomes in even the most severely injured patients with complex extremity injuries, but salvage is no guarantee of functionality. As for the mangled limb in these patients, it is unlikely a scoring system will allow a clear cut-off point for amputation versus salvage. Because of this the decision between amputation or limb salvage needs to be made on an individual case basis, taking into account the characteristics of the patient, the experience of the team, the technology available in the hospital, and the real possibility of saving the function of the limb knowing that and early amputation should not be considered a treatment failure but rather a means of meeting goals of treatment.

\section{Summary}

Prevention of wound infection and sepsis remains the prime objective in the management of open tibial fractures. The reported infection rates in these fractures range from 3 to $25 \%$ and are a challenge to every surgeon who treats them. The cooperation between orthopaedic and plastic surgeons together with the use of modern surgical techniques and wound management has resulted in a dramatic reduction in infection rates. The principles for treating an open fracture include: resuscitation, initial recognition of the severity of the injury, analgesia, anti-tetanus therapy, antibiotics therapy; debridement and irrigation, stabilization of the fracture, possibly use of adjuncts such as antibiotic beads and vacuum therapy, and early soft tissue coverage with well vascularized tissue when indicated. Classifying an open fracture in the emergency room is useful for physician-to-physician communication as well as for deciding the management of the initial injury. However the injury must be re-classified at the end of the initial debridement. The Gustilo and Anderson classification has been the gold standard to help classify these injuries for many years because of its simplicity and familiarity. We believe however that in future years a new classification system such as the OTA classification could replace this system and became the new gold standard. Treatment should follow the $\mathrm{BOA} / B A P R A S$ guidelines. ${ }^{2}$ Pulsatile lavage systems are unwarranted. A simple brush-suction irrigation plus bulb syringe removal in organic contamination have shown no less effectiveness that HPPL. Adequate debridement is the single most important factor in the attainment of a good result in the treatment of an open fracture, most studies have indicated that time to debridement is not an independent predictor of infection for open fractures. Of greater importance is time to admission and the early administration of antibiotics. The timing of debridement should therefore be determined by adequacy of resuscitation, presence of appropriate and alert staff, and operating room availability. Early stabilization of the fracture is essential when managing an open tibia fracture and current practice indicates this should be done at the time of initial surgical debridement if at all possible. The technique of early stabilization remains a controversial topic, and should be selected according to the fractures pattern, contamination and bone loss. Spanning external fixators can be a convenient modality in most cases for initial stabilization. They should be exchanged to internal fixation as soon as the soft tissues allow. Initial Internal fixation is safe if there is minimal contamination and soft tissue coverage is achievable at the same time as insertion of the implant. Modern multi-planar and circular fixators must be used if there is significant contamination, bone loss and multilevel fractures of the tibia. Evaluate the possible need for adjuncts such as vacuum therapy and antibiotic beads especially in cases with high degree of contamination and bone loss. However it is important to remember that vacuum therapy and antibiotic beads are adjuncts and are not a replacement for meticulous wound toilet and debridement of necrotic/ contaminated tissue and appropriate soft tissue coverage whether that be primary closure with sutures or a local or free musculocutaneous skin flap. It is always important to remember that saving a limb without good function can be much worse than having an early amputation and limb fitting rehabilitation.

\section{Acknowledgments}

None.

\section{Conflicts of interest}

None. 


\section{References}

1. Gustilo RB, Anderson JT Prevention of infection in the treatment of one thousand and twenty-five open fractures of long bones: retrospective and prospective analyses. J Bone Joint Surg Am. 1976;58(4):453-458.

2. The British Orthopaedic Association and British Association of Plastic, Reconstructive and Aesthetic Surgeons (BOA/BAPRAS). 2009Standards for treatment of open fractures of the lower leg. BMJ 339: b5092.

3. Puno RM, Teynor JT, Nagano J et al. Critical analysis of results of treatment of 201 tibial shaft fractures. Clin Orthop Relat Res2. 1986;12:113-121.

4. Harley JB, Beaupre LA, Jones CA et al. The effect of time to definitive treatment on the rate of nonunion and infection in open fractures. $J$ Orthop trauma. 2002;16(7):484-490.

5. Draeger RW, Dirschl DR, Dahners LE Debridement of cancellous bone: A comparison of irrigation methods. J Orthop Trauma. 2006;20(10):692-698.

6. Anglen JO Comparison of soap and antibiotic solutions for irrigation of lower-limb open fracture wounds. A prospective, randomized study. $J$ Bone Joint Surg Am 2005;87(7):1415-1422.

7. Orthopaedic Trauma Association: Open Fracture Study Group A new classification scheme for open fractures. $J$ Orthop Trauma. 2010;24(8):457-465.

8. Klasen HJ A review on the nonoperative removal of necrotic tissue from burn wounds. Burns. 2000;26(3):207-202

9. Bhandari M, Schemitsch EH, Adili A et al. High and low pressure pulsatile lavage of contaminated tibial fractures: an in vitro study of bacterial adherence and bone damage. J Orthop Trauma. 1999;13(8):526-533.

10. Dirschl DR, Duff GP, Dahners LE et al. High pressure pulsatile lavage irrigation of intraarticular fractures: effects on fracture healing. J Orthop Trauma. 1998;12(7):460-463.

11. Lee EW, Dirschl DR, Duff G et al. High-pressure pulsatile lavage irrigation of fresh intraarticular fractures: effectiveness at removing particulate matter from bone. J Orthop Trauma. 2002;16(3):162-165.
12. Polzin B, Elllis T, Dirschl DR Effects of varying pulsatile lavage pressure on cancellous bone structure and fracture healing. JOrthop Trauma. 2006;20(4):261-266.

13. Skaggs DL, Friend L, Alman B et al. The effect of surgical delay on acute infection following 554 open fractures in children. $J$ Bone Joint Surg Am. 2005;87(1):8-12.

14. Giannoudis PV, Papakostidis C, Roberts C A review of the management of open fractures of the tibia and femur. J Bone Joint Surg Br. 2006;88(3):281-289.

15. Xu X, Li X, Liu L A meta-analysis of external fixator versus intramedullary nails for open tibia fracture fixation. J Orthop Surg Res. 2014;9:75.

16. Rommens P, Van Raemdonck D, Broos P Reosteosynthesis of the tibial shaft. Part I. Changement of procedure after external fixation. Acta Chir Belg. 1989;89(5):281-286.

17. Inan M, Halici M, Ayan I et al. Treatment of type IIIA open fractures of tibial shaft with Ilizarov external fixator versus unreamed tibial nailing. Arch Orthop Trauma Surg. 2007;127(8):617-623.

18. Schenker ML, Yannascoli S, Baldwin KD et al. Does timing to operative debridement affect infectious complications in open long-bone fractures? A systematic review. J Bone Joint Surg Am. 2012;94(12):1057-1064.

19. Rios Luna A, Fahandezh-Saddi H, Villanueva Martinez M, Lopez AG Oct-Dec Pearls and tips in coverage of the tibia after a high energy trauma. Indian J Orthop. 2008;42(4):387-394.

20. Bhandari M, Guyatt GH, Swiontkowski MF et al. Treatment of open fractures of the shaft of the tibia: a systematic overview and metaanalysis. J Bone Joint Surg Br. 2001;83(1):62-68.

21. Fadhli A, Alexander G, Kanjor JR Versatile use of vacuum-assisted healing in fifty patients. Indian J Plast Surg. 2009;42(2):161-168.

22. Shanmuganathan $\mathrm{R}$ The utility of scores in the decision to salvage or amputation in severely injured limbs. Indian Journal of Orthopaedics. 2008;42(4):368-376.

23. Prasarn ML, Helfet DL, Kloen $P$ Management of the mangled extremity. Strategies in Trauma and LimbReconstruction. 2012;7(2):57-66. 